\title{
Umweltanalyse mit GIS und Fernerkundung - Einleitung zum Themenheft
}

Geographische Informationssysteme (GIS) und Fernerkundung sind neue Technologien, die in den vergangenen Jahrzehnten enorme Entwicklungen durchgemacht und in der Forschung und der Öffentlichkeit besonders in den letzten Jahren eine breite Akzeptanz gefunden haben. Es sind dies Werkzeuge der räumlichen Informationsverarbeitung, die über traditionelle Disziplinengrenzen hinweg für eine Vielzahl von Aufgaben eingesetzt werden können. Neben Anwendungen vom Ingenieurbereich über die Fahrzeugnavigation bis hin zu überregionalen sozioökonomischen Analysen sind diese Technologien insbesondere für Aufgaben im Bereich der Umweltforschung und des Umweltmanagements einsetzbar. Der Fernerkundung mit ihrer Vielfalt an Sensorsystemen, Plattformen und Aufbereitungsverfahren kommt dabei vor allem die wichtige Aufgabe der flächendeckenden, zeitgerechten Erfassung und problemgerechten Vorverarbeitung und Interpretation von Erdbeobachtungsdaten $\mathrm{zu}$, während Geographische Informationssysteme eine allgemeine Plattform für die Verknüpfung vielfältiger Informationsquellen sowie die Speicherung, Manipulation, Analyse und graphische Präsentation raumbezogener Daten zur Verfügung stellen. Im Bereich der Datenverwaltung, Datenmanipulation und Visualisierung verzahnen sich die beiden Fachbereiche immer mehr und wachsen langsam zusammen. In beiden Bereichen sind einerseits Bemühungen zur Entwicklung von grundlegenden Werkzeugen (Erfassungs- und Interpretationstechniken, Datenmodellierung, Analyse- und Darstellungsmethoden) und andererseits die Erforschung von Anwendungsstrategien in verschiedenen umweltrelevanten Fachgebieten erforderlich. Die Geographie leistet zu diesen Entwicklungen in einem interdisziplinären Umfeld gemeinsam mit Fachgebieten wie der Geodäsie, Photogrammetrie, Informatik, den Bildwissenschaften, der Kartographie, den verschiedenen Geowissenschaften, der Planung und vielen anderen ihren wichtigen Beitrag.

GIS und Fernerkundung sind Wachstumsbereiche sowohl bezüglich der Zahl der installierten Systeme (lokale, regionale, nationale und internationale Verwaltungsstellen; Private) als auch im Bereich der Grundlagen- und angewandten Forschung. Dies äußert sich unter anderem auch darin, daß die internationalen Dachverbände der beteiligten Fachbereiche (International Geographical Union IGU, International Cartographic Association ICA, International Society of Photogrammetry and Remote Sensing ISPRS, Societé Internationale de Géo- mètres SIG usw.) Fachkommissionen unterhalten, die sich mit der Technologie der räumlichen Datenbeschaffung und Datenanalyse auseinandersetzen. Europaweit wurde eine Dachorganisation für Geoinformation (European Umbrella Organisation for Geographical Information EUROGI) gegründet, der auch eine schweizerische Zweiggesellschaft (Schweiz. Organisation für Geo-Information SOGI) angehört. Zudem hat die European Science Foundation (ESF) in den Jahren 1993-1997 zur Förderung der technologischen Entwicklung und der internationalen Zusammenarbeit ein Forschungsprogramm lanciert (Geographic Information Systems Data Integration and Data Base Design GISDATA), das verschiedene Forschungsaktivitäten nach sich gezogen hat. Von all diesen Institutionen sowie auch von kommerziellen Softwareanbietern im GIS- und Fernerkundungsbereich werden immer wieder Konferenzen, Workshops und Fachausstellungen organisiert.

So hat sich auch die ASG (Association Suisse de Géographie/Verband Geographie Schweiz) entschlossen, im Herbst 1996 ihr Fachsymposium anläßlich der Jahrestagung der Schweizerischen Akademie der Naturwissenschaften (SANW) dem Problemkreis «Einsatz von Geographischen Informationssystemen und Fernerkundung in der Umweltanalyse» zu widmen. Dieser Anlaß wurde gemeinsam mit der Schweizerischen Kommission für Fernerkundung (SKF) der SANW und der "Interuniversitären Partnerschaft Erdbeobachtung und Geoinformatik» der Universität und ETH Zürich durchgeführt und vom Geographischen Institut der Universität Zürich organisiert. Dieses Symposium sollte vor allem jungen Forscherinnen und Forschern sowie Praktikern aus der Schweiz im Bereich der GIS-Anwendung eine Plattform bieten, um ihre Projekte zu präsentieren. Als Zielpublikum waren Naturwissenschafter/-innen verschiedener Fachrichtungen, aber auch breite Interessentenkreise aus Verwaltung, der Privatwirtschaft und dem Schulbereich angesprochen. Dieser Anlaß wurde denn auch von rund hundert Personen besucht. Die insgesamt dreizehn Fachvorträge sind in einem Tagungsband veröffentlicht wor-

Kurt Brassel, Prof. Dr., Geographisches Institut der Universität Zürich, Winterthurerstraße 190, 8057 Zürich

kbrass@geo.unizh.ch 
den (BRASSEL et al. 1996). Es waren dies Referate von zwei eingeladenen Hauptreferenten sowie eine Reihe von Beiträgen, die aufgrund einer offenen Ausschreibung eingegangen sind. Thematisch erstreckte sich das Spektrum von Übersichtsartikeln aus der Sicht der Forschung und der öffentlichen Verwaltung zu Projektberichten aus den Themenbereichen Umweltdaten und Speicherstrategien, GIS-gestützte Fernerkundungsprojekte, Waldbrandmodellierung, GIS in der Zoologie und GIS in der Pedologie. Zudem hat sich die Redaktion der "Geographica Helvetica» bereit erklärt, eine Auswahl von Beiträgen in z. T. neugefaßter und ergänzter Form in diesem Themenheft «Umweltanalyse mit GIS und Fernerkundung» herauszugeben. Nachdem ein erster Hauptvortrag des Symposiums bereits in der Nr. 4/1996 (HUMBEL 1996) veröffentlicht worden ist, publizieren wir in diesem Themenheft das zweite Gastreferat und drei Anwendungsberichte.

Prof. Manfred Ehlers von der Universität Vechta (Niedersachsen, BRD) gilt als Spezialist, der in Lehre und Forschung sowohl Fernerkundung als auch Geographische Informationsverarbeitung vertritt. In seinem Beitrag zur Rolle dieser beiden Fachgebiete im Umweltmonitoring und Umweltmanagement diskutiert er Forschungsansätze zur Integration der beiden Disziplinen und leitet Ansprüche für nachhaltige Planungskonzepte ab.

C. Ehrler und J. Schaper berichten über eine Analyse von Ausaperungsmustern der saisonalen Schneedecke, die mit Hilfe von Fernerkundungs- und GIS-Methoden durchgeführt worden ist. Insbesondere werden Zusammenhänge zwischen der Schneebedeckung und Jahreszeit, Höhenlage, Exposition, Geländeneigung und Beschaffenheit der Schneeunterlage in Teilen der Schweizer Alpen untersucht.

In ihrem Beitrag kombinieren P. Pirchl, P. Hirtz, M. Suter und D. Nüesch Bilddaten verschiedener Sensoren und unterschiedlicher Auflösung mit Gelände- und Landnutzungsinformation mit dem Ziel, möglichst realistische perspektivische Landschaftsvisualisierungen zu erzielen. Dabei setzen sie Methoden der Fernerkundung, der Bildverarbeitung und Computergraphik sowie Geographische Informationssysteme ein.

Der letzte Beitrag von A. Bachmann, R. Schöning und B. Allgöwer zeigt die Anwendungsmöglichkeiten von Geographischen Informationssystemen für die Analyse und das Management von Wald- und Buschbränden. Die vorgestellten Konzepte verwenden eine breite Palette von raumkodierten Umweltvariablen, die mit Hilfe von Geographischen Informationssystemen modelliert werden. Ziel dieses durch die Wissenschaftliche Nationalparkkommission (WNPK) und ein EU-Projekt mitfinanzierten Forschungsprojektes ist ein GIS-basiertes Entscheidungsunterstützungssystem für ein kontrolliertes Waldbrandmanagement.

Diese Auswahl von Anwendungsprojekten soll die vielseitigen Einsatzmöglichkeiten der Fernerkundungs- und GIS-Technologien andeuten. Vor allem wird auch demonstriert, daß nach Jahrzehnten der Forschungstätigkeit diese Technologien allmählich für den realen Einsatz im Umweltmanagement heranreifen.

\section{Literatur}

BRASSEL, K., ITTEN, K., SCHMITT, E. (Hrsg.) (1996): Einsatz von Geographischen Informationssystemen und Fernerkundung in der Umweltanalyse, Tagungsband zum Symposium der 176. Jahresversammlung der SANW, GeoprocessingReihe, Vol. 31, Geographisches Institut der Universität Zürich. HUMBEL, R. (1996): Die Bundesstatistik als Anbieter und Nutzer von raumbezogenen Daten, Geographica Helvetica, 51. Jg., Nr. 4, S. 169-179. 